\title{
Flowability of lignocellusic biomass powders: influence of torrefaction intensity
}

\author{
John Pachón-Morales", Julien Colin, Floran Pierre, Thibaut Champavert, François Puel and Patrick Perré \\ LGPM, Centrale Supélec, Université Paris-Saclay, Grande Voie des Vignes, 92295 Châtenay-Malabry, France
}

\begin{abstract}
The poor flowability of powders produced from raw lignocellulosic biomass may be an economically issue for the production of second-generation biofuels. Torrefaction is a pre-treatment step of the gasification process that improves the physical characteristics of biomass by making it more coal-like. Particularly, the loss of resilience allows a reduction of the grinding energy consumption and is likely to improve the flow behaviour of woody powders. In this study, we investigated the effect of particle size and shape distribution on flow properties (unconfined yield stress and flowability factor) of powder from raw and torrefied biomass (Picea abies). Several intensities of torrefaction were tested, and its extent was quantified by the global mass loss, chosen as synthetic indicator of torrefaction intensity (its accounts for both the temperature level and the residence time). The intensity of torrefaction shifts the particle size distribution towards smaller sizes. An effect on the circularity and aspect ratio was also observed. A strong, positive correlation was obtained between the measured flowability of biomass powders at different consolidation stresses and the intensity of heat treatment. These results confirm the interest of torrefaction as a pre-treatment step and aim to provide new knowledge on rheological properties of biomass powders.
\end{abstract}

\section{Introduction}

In spite of the recent drop of oil price, the development of fuel from renewable resources is absolutely required to face global warming. After the first generation of biofuels that consumes edible products, a second generation using the lignocellulosic part of plants - such as wood -, is yet to be developed at the industrial scale. One possible $B t L$ (biomass to liquid) route includes a gasification process followed by a Fischer-Tropsch synthesis. Most of gasification processes require a supply of ground biomass in the form of powder for three main reasons: to ease the conveying of the raw material along the processing chain, to optimize its injection into the gasifier and to increase the chemical reactivity of the biomass. Currently, biomass transport is a key obstacle for the cost-competitive production of second-generation biofuels. Indeed, due to its fibrous structure, the grinding of native biomass is an energy intensive process and the powder obtained does not flow well [1]. Thus, a pre-treatment step, such as torrefaction, is needed to improve the process.

Torrefaction is a thermal treatment operating at moderate temperature $\left(200\right.$ to $\left.300^{\circ} \mathrm{C}\right)$ under atmospheric pressure in absence of oxygen. The process is traditionally characterised by low particle heating rates $\left(<50{ }^{\circ} \mathrm{C} \cdot \mathrm{min}^{-1}\right)$ and by a relatively long reactor residence time (typically higher than 30 minutes) [2]. The resulting product is intermediate between wood and charcoal and exhibits several advantages when compared to the original material such as a decreased hygroscopicity, a dimensional stabilization, an increased resistance to biological decay, a loss of mechanical resilience, etc. [3]
Several researchers have studied the question of estimating biomass flowability. Nevertheless, despite the frequent occurrence of handling problems, there is still a relatively poor knowledge of the flow properties of biomass materials [1]. More research is therefore needed in this field to ensure, the sustainability of the supply chain [4]. This includes a better understanding of the flowability of lignocellulosic biomass powders, namely the combined effect of the torrefaction and grinding parameters. This is needed for engineers to develop costeffective storing, conveying and processing strategies.

In this study, we investigate the effect of different torrefaction intensities on the flow properties of ground biomass, and assess the relations between size and shape of the particles and the flow behaviour of these powders.

\section{Materials and methods}

\subsection{Material preparation}

\subsubsection{Wood Chip preparation}

A spruce (Picea abies) tree from a plantation in the forest of Le Châtaignier in Riotord (France) was used in this work.

All samples came from the same board. The size of the samples was chosen to fulfil several constraints such as the amount required for flowability tests (width), the grinding capacity of the grinder (maximum length) and the homogeneity of the heat treatment (small thickness). The size of each individual wood chip was thus fixed to

\footnotetext{
* Corresponding author: john.pachon@centralesupelec.fr
} 
$20 \times 20 \times 3 \mathrm{~mm}^{3}$. Each sample number of Table 1 is composed of 32 chips of this size.

As it is well-known that the wood properties may vary in the tangential and radial directions, the 32 wood chips of each sample are selected following a recurrent stepped scheme along the board in order to average this variability and to obtain twin samples (Fig. 1).

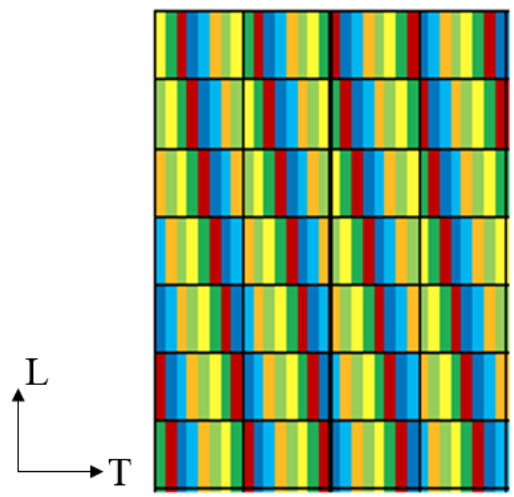

Fig. 1. Stepped cutting plan of the spruce board (Each colour corresponds to one individual sample of 32 chips). L: longitudinal direction, $\mathrm{T}$ : tangential direction.

\subsubsection{Torrefaction procedure}

A batch torrefaction device specifically developed in our laboratory was used to treat our sample [5]. The atmosphere of a hermetically closed Memmert UFP400 chamber was controlled by a nitrogen flow rate $\left(5 \mathrm{~L} . \mathrm{min}^{-}\right.$ $\left.{ }^{1}\right)$ to reduce the oxygen content, thereby avoiding oxidation and ignition. In all cases, the oxygen level remained below $1.5 \%$. A powerful fan inside the chamber insures an efficient heat transfer either to heat-up the sample or to limit thermal overshot due to exothermic reactions. The gas temperature was measured nearby the samples using a calibrated thermocouple.

A total of 6 treatments were conducted at 220,250 and $280{ }^{\circ} \mathrm{C}$ for 1 or 5 hours according to the following protocol: a. heating from room temperature (about $20^{\circ} \mathrm{C}$ ) at a rate of $5^{\circ} \mathrm{C} \min ^{-1}$ up to $100{ }^{\circ} \mathrm{C}$. b. plateau at $100^{\circ} \mathrm{C}$ for 30 minutes in order to evaporate the residual bound water before the heat treatment step. c. heating at a rate of $5^{\circ} \mathrm{C} \mathrm{m^{-1 }}$ until the treatment temperature. d. plateau at the treatment temperature for the desired duration and e. cooling down thanks to thermal losses and the nitrogen flow entering the reactor.

After treatment, samples were weighed to determine the mass loss. Mass loss $(M L)$ due to heat treatment was calculated as follows:

$$
M L(\%)=\frac{m_{0}-m_{t}}{m_{0}} \cdot 100
$$

Where, $m_{0}$ and $m_{t}$ are the oven-dry mass before and after torrefaction, respectively. Mass loss is known to be a good indicator of the treatment intensity [2].
Table 1. Heat treatment intensities.

\begin{tabular}{cccc}
\hline $\begin{array}{c}\text { Sample } \\
\text { number }\end{array}$ & $\begin{array}{c}\text { Treatment } \\
\text { temperature } \\
\left({ }^{\circ} \mathbf{C}\right)\end{array}$ & $\begin{array}{c}\text { Duration of } \\
\text { treatment } \\
\text { (h) }\end{array}$ & $\begin{array}{c}\text { Mass loss } \\
\mathbf{( \% )}\end{array}$ \\
\hline 1 & 220 & 1 & 11,4 \\
2 & 220 & 5 & 14,7 \\
3 & 250 & 1 & 17,4 \\
4 & 250 & 5 & 25,4 \\
5 & 280 & 1 & 30,9 \\
6 & 280 & 5 & 46,5 \\
7 & \multicolumn{2}{c}{ Non-treated } & 0 \\
\hline
\end{tabular}

\subsubsection{Grinding procedure}

Heat-treated samples were ground in a M20 IKA semiautomatic laboratory $550 \mathrm{~W}$ knife miller. The rotational speed of the knives was $20000 \mathrm{rpm}$. The same grinding protocol was applied to all of the samples: a $95 \mathrm{~s}$ grinding made in several cycles (13) with intermediate waiting times of $15 \mathrm{~s}$ in order to avoid any additive heat treatment due to thermal dissipation. A circulation of cold water was applied to reduce heating during grinding.

\subsection{Morphological granulometry}

A Sympatec-QICPIC morphological granulometer with a M7 lens (measuring range from 10 to $10240 \mu \mathrm{m}$ ) with a GRADIS dispersion and VIBRI feeding units was used in this work [7]. To conduct the test, a sample of about $5 \mathrm{~g}$ of wood powder was loaded onto the hopper of the instrument. The size and shape of powders were recorded and analysed by the manufacturer software.

The Feret mean diameter, the mean value of the distance of two tangents to the particle contour over several orientations was measured: The mean diameter of the sample is then calculated as:

$$
d_{p}(1,0)=\left(\sum_{i=1}^{n} n_{i} d_{i}\right) /\left(\sum_{i=1}^{n} n_{i}\right)
$$

Where $n_{i}$ is the number of particles with a Feret mean diameter $d_{i}$. Shape of the particles is characterised through the circularity and the aspect ratio. For the current study, circularity, $\psi_{S}$ (from 0 to 1 ), is defined as the ratio of the perimeter of a circle having the same area as the projected area of a particle to its actual perimeter. A perfect sphere would give $\psi_{S}=1$ and the smaller the value of $\psi_{S}$, the more irregular is the shape of the particle. The aspect ratio, the ratio of the minimal to the maximal Feret diameter, gives an indication of the elongation of the particle.

\subsection{Flow properties testing}

A RST-XS Schulze ring shear tester was used to assess the flow properties of biomass powders. The ring shear tester is a widely used device to measure flow properties of powders, including angle of internal friction, wall friction and bulk density. The standard procedure leads to results with low variability [6]. 
A set of three pre-consolidation stresses $\left(\sigma_{\text {pre }}\right)$, considered as representative of the stress range for industrial applications, were tested: 2,5 and $10 \mathrm{kPa}$ [12]. Three shear points at 25,50 and $75 \%$ of $\sigma_{\text {pre }}$ for each consolidation stress were used to determine the yield locus of each sample. Using Mohr circle analysis, the yield loci obtained allowed to evaluate the consolidation stress $\sigma_{l}$ and the unconfined yield strength $\sigma_{c}$ for the different normal stresses at preshear applied. The ratio $F F C$ (flow function coefficient) of consolidation stress to unconfined stress $\left(\sigma_{l} / \sigma_{c}\right)$ is used to characterize flowability numerically.

The larger $F F C$ is, i.e., the smaller the ratio of the unconfined stress to the consolidation stress, the better a bulk solid flows.

\section{Results and discussion}

\subsection{Particle Size distribution}

In Fig. 2 the cumulative size distributions of the powder samples are represented. The intensity of heat treatment, represented by the mass loss of the samples, has an obvious impact on the size distribution of the biomass powders: the loss of resilience allows to obtain smaller particles by grinding.

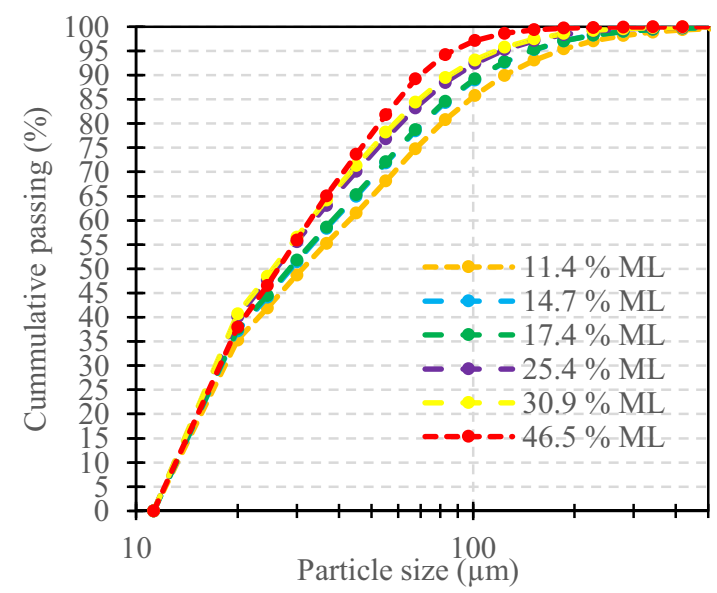

Fig. 2. Cumulative passing at several torrefaction intensities.

The reduction of the resilience at increasing intensity of heat treatment makes the samples brittle, thus the particle size distribution shifts towards smaller particle sizes and its dispersity is reduced. Similar conclusions have been also observed from other studies, for different kinds of biomass powders and torrefaction conditions [10], [11], [8].

Fig. 3 shows the value of the mean diameter and the cumulative percentage of particles with a Feret mean diameter below $101.2 \mu \mathrm{m}$ at various torrefaction intensities. The cumulative number represents the fraction of particles having a diameter of $101.2 \mu \mathrm{m}$ or less.

The mean diameter decreases dramatically whereas $\mathrm{d}_{101.2}$ increases with the increase of torrefaction intensity, the grinding conditions remaining the same for all the samples. This shows that there is an improvement in the grindability characteristics of treated biomass. This result is in good agreement with those presented in previous works [8], [9].

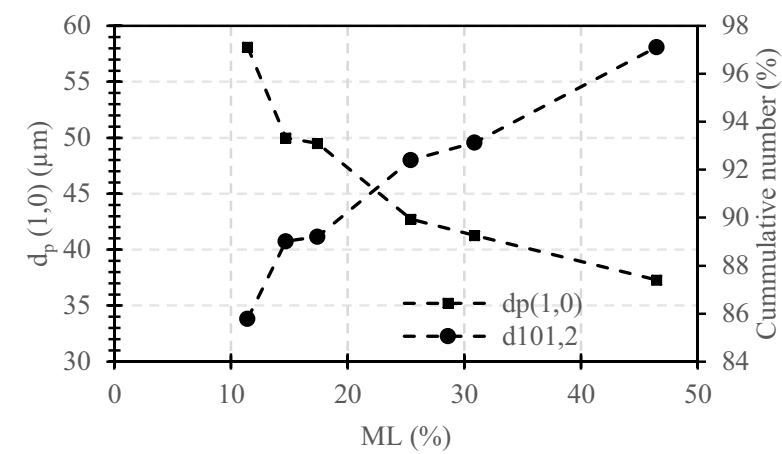

Fig. 3. Effect of intensity of heat treatment on the mean diameter and cumulative percentage of particles with a size below $101.2 \mu \mathrm{m}$.
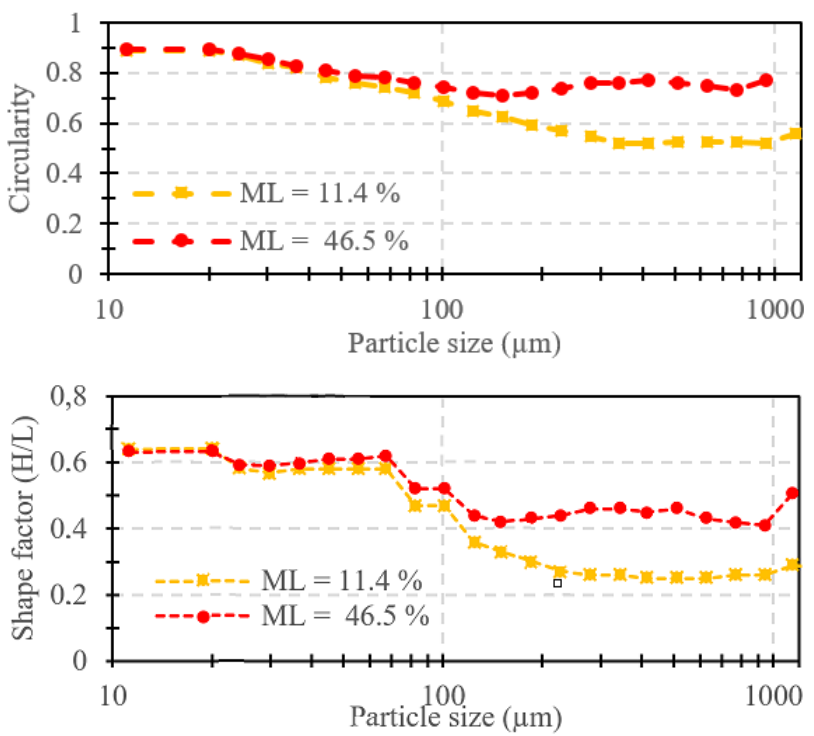

Fig. 4. Shape of the treated spruce powders at two different torrefaction intensities.

Fig. 4 represents the morphological parameters of the biomass powders for two torrefaction intensities. On the whole, circularity of spruce powders was improved as the intensity of treatment increased from $M L=11.4 \%$ to $M L=46.5 \%$ for particles size above $50 \mu \mathrm{m}$. More spherical particles are thus globally obtained when a severe treatment is applied $\left(280^{\circ} \mathrm{C}, 5 \mathrm{~h}\right)$. This denotes a loss of the fibrous behaviour. However, no significant difference in circularity of ground biomass was observed for torrefaction intensities from $11.4 \%$ to $30.9 \%$. It seems that the finer particles tend to be more spherical. Nevertheless, a more sensitive morphological analysis is necessary in order to better characterize these small particles $\left(d_{p}<100 \mu \mathrm{m}\right)$. Similar conclusions can be drawn regarding the shape factor: more uniform particles are obtained when intensity of torrefaction is increased.

\subsection{Flowability}

Fig. 5 presents the flow functions for different torrefaction intensities along with the flowability regimes from not flowing to free-flowing behaviour. A clear 
dependence between the extent of the heat treatment and the flowability of spruce powders is depicted. The native ground biomass has a cohesive behaviour that decreases with the heat treatment until a nearly free flowing feature for the most intensively treated sample. In almost all cases, the unconfined yield stress increases with the consolidation stress to the same extent, so the regime of flowability for the powders is independent of the consolidation stress applied (except for the most intensely treated sample for which a free-flowing behaviour is reached at elevated consolidation stresses).

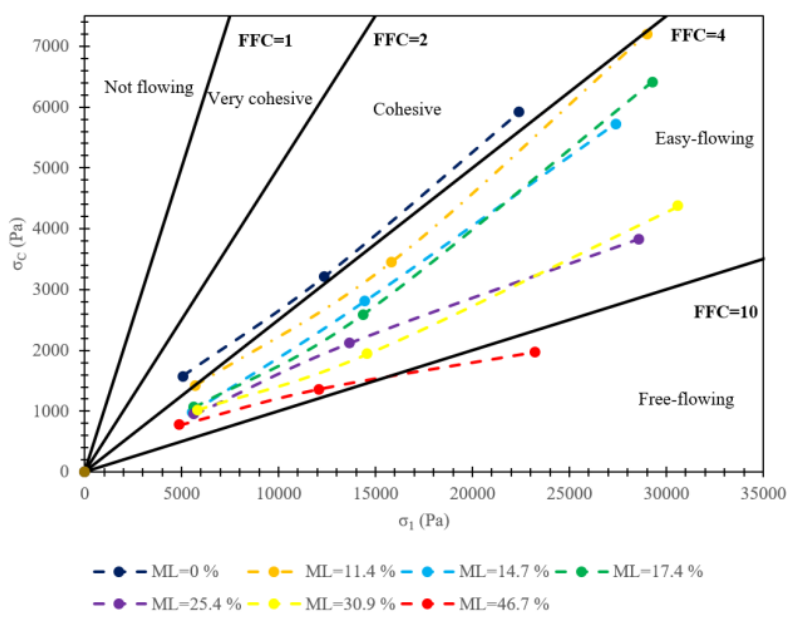

Fig. 5. Instantaneous flow functions for several intensities of torrefaction and lines of constant flowability FFC (solid lines).

For all samples, better flowability is obtained at a greater consolidation stress but there is no influence of the stress level on the qualitative ranking of the set of powders. This last feature is in good agreement with the general behaviour for most of the powders reported in literature [12].

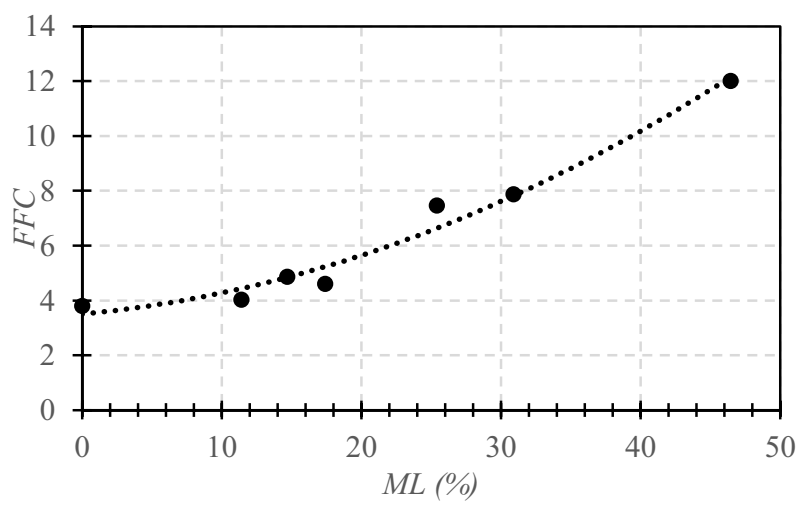

Fig. 6. Effect of torrefaction intensity on the flowability factor of the biomass powders. $\sigma_{\text {pre }}=10 \mathrm{kPa}$.

A direct relationship between the intensity of torrefaction and the flowability factor was found (Fig. 6). For sake of clarity, only the values at $\sigma_{p r e}=10 \mathrm{kPa}$ are presented, but similar tendencies were verified for the others values. The improvement of flow behaviour with the extent of heat treatment could be seen as a combined effect of several factors: the modifications on particle size and width of the distribution, the improvement of shape characteristics and the modification of the surface interactions. Further stage of our study will consist of isolating each factor in order to discriminate their effects on flowability. The lower values of circularity and larger aspect ratios lead to more frequent interparticle entanglements, which degrades flowability. These findings are in agreement with literature reports [13], [14].

\section{Conclusions}

This work evaluated the impact of six operating conditions of torrefaction (from $11.4 \%$ to $46.5 \%$ of mass loss) on the flowability of a biomass powder measured in a ring shear tester, as well as on particles size distribution and morphology. Several tendencies are clearly drawn from this work:

- the particle size distribution shifts towards smaller particle sizes and its dispersity is reduced with increase in torrefaction intensity,

- due to the loss of the fibrous behaviour, the circularity of spruce powders is improved at high-treatment intensities,

- an improvement of the flowability of spruce powders by heat treatment is clearly exhibited. This behaviour gradually shifts from a cohesive behaviour for the native biomass to a nearly free flowing behaviour for a heat treatment with $46.5 \%$ of mass loss.

\section{References}

[1] Miccio, F., Barletta, D., \& Poletto, M. (2013). Pow. Tech, 312-321.

[2] Bergman, P., Boersma, A., Zwart, R., \& Kiel, J. (2005). Petten, The Netherlands: ECN.

[3] Almeida, G., Brito, J. O., \& Perré, P. (2010). Bio. tech. 9778-9784.

[4] Ramirez-Gomez, A. (2016). Part. sci. and tech..

[5] Colin, J. (2007). U. H. Poincaré and AgroParisTech.

[6] ASTM D6773. (2002). ASTM International.

[7] Sympatec GmbH. (2016). Laser Diffraction Sensors. Retrieved from http://www.sympatec.com

[8] Arias, B., Pevida, C., Fermoso, J., Plaza, M., Rubiera, F., \& Pis, J. (2008). Fuel pro. tech., 169-75.

[9] Phanphanich, S., Mani, S. (2011). Bior. tech., 12461243.

[10] Himmel, M., Tucker, M., Baker, J., Rivard, C., \& Grohmann, K. (1985). Biotech. and bioeng. symp., 39-58. [11] Mani, S., Tabil, L. G., \& Sokhansanj, S. (2006). Biom. and bioen. 30, 648-654.

[12] Schulze, D. (2007). Powders and bulk solids. Braunschweig: Springer.

[13] Crawford, N., Ray, A., Yancey, N., \& Nagle, N. (2015). Fuel pro. tech., 46-56.

[14] Almendros, M., Bonnefoy, O., Govin, A., Nastoll, W., \& Sanz,. 19th European Biomass Conference 2011. Berlin. 